\title{
Better Functional Outcome and Pain Relief in the Far-Lateral-Outside-in Percutaneous Endoscopic Transforaminal Discectomy
}

\author{
Sheng-Fen Wang ${ }^{1, *}$ \\ Shih-Feng Hung ${ }^{2, *}$ \\ Tsung-Ting Tsai ${ }^{2}$ \\ Yun-Da Li ${ }^{2}$ \\ Ping-Yeh Chiu ${ }^{2}$ \\ Ming-Kai Hsieh ${ }^{2}$ \\ Fu-Cheng $\mathrm{KaO}^{2,3}$
}

'Department of Anesthesiology, Chang Gung Memorial Hospital and Chang Gung University College of Medicine, Taoyuan, Taiwan; ${ }^{2}$ Department of Orthopaedic Surgery, Spine Section, Chang Gung Memorial Hospital and Chang Gung University College of Medicine, Taoyuan, Taiwan; ${ }^{3}$ Institute of Biomedical Engineering, National Tsing Hua University, Hsinchu, Taiwan

*These authors contributed equally to this work
Correspondence: Fu-Cheng Kao Department of Orthopaedic Surgery, Spine Section, Chang Gung Memorial Hospital and Chang Gung University College of Medicine, No. 5, Fusing St., Gueishan, Taoyuan, 333, Taiwan

Tel +886-3-328I200 ext. 3612

Fax +886-3-3278। I3

Email afogi73@gmail.com
Background: Even though percutaneous endoscopic transforaminal discectomy (PETD) has been widely used for the surgical treatment of lumbar herniated disc, there are only a few studies directly comparing the clinical outcomes between microdiscectomy (MD) and PETD. Therefore, further studies are needed in order to compare the clinical outcomes between PETD and MD on a single level more thoroughly.

Methods: We proposed a far-lateral-outside-in technique for PETD to get an entry point without complex planning and facet violation. From September 2017 to September 2019, a total of 155 patients (69 with PETD and 86 with MD) were enrolled for this retrospective study, with the inclusion criteria: single level between L2 and S1, clinical sciatica for at least 6 weeks, and failed attempts with non-surgical treatments. Patients were excluded due to cauda equina syndrome, progressive neurologic deficits, history of lumbar spinal surgery, endplate modic changes, severe disc degeneration and less than 24 months of follow-up.

Results: PETD showed significantly shorter surgery time and less intraoperative blood loss compared to MD. The difference between PETD and MD in VAS back pain (but not VAS leg pain) at follow-up time was significant. The ODI improvement in the follow-up time of 6 months between the 2 groups showed a significant difference (PETD: $31.23 \pm 6.59$, MD: $39.85 \pm 7.81, \mathrm{p}<0.001)$. MD had a significantly higher chance of postoperative wound infection and poor healing, while PETD was more likely to have recurrence of the herniated disc.

Conclusion: MD has been the gold standard procedure for LDH. However, with the advanced endoscopic technology and surgical technique, PETD has emerged as an alternative method due to higher functional outcome and less wound problems.

Keywords: microdiscectomy, PETD, discectomy

\section{Introduction}

Lumbar disc herniation (LDH) is one of the most common causes of lower back pain associated with sciatica, affecting people aged 35 to $50 .^{1,2}$ Despite being a highly prevalent disease, $\mathrm{LDH}$ can be asymptomatic and mostly require only conservative treatment. ${ }^{3}$ However, in around $15 \%$ of these patients, surgical intervention is recommended owing to failure on conservative therapy. ${ }^{4}$ Among surgical treatments, microdiscectomy (MD) is generally acknowledged as the gold standard procedure for symptomatic $\mathrm{LDH}^{5}$ Meanwhile, with the advance in surgical technology, many minimally invasive techniques have been developed and emerged as alternative methods for symptomatic LDH. ${ }^{6,7}$ 
Percutaneous endoscopic transforaminal discectomy (PETD) was first introduced in the late twentieth century by Kambin et al and has become one of the minimally invasive approaches to $\mathrm{LDH} .{ }^{8}$ Thanks to the development of a navigation system and optical technology, the indications of PETD have expanded drastically in a way that PETD could literally be equivalent to MD. ${ }^{9}$ There are two major techniques of PETD. ${ }^{10-12}$ The earlier one is intradiscal, inside-out approach and the more recent approach is outside-in approach. It is difficult for the intradiscal, inside-out approach to remove migrated intra- and extraforaminal LDH meanwhile ensuring thorough nerve root decompression intraoperatively. Moreover, the anatomic structure could hardly be preserved in such approach. ${ }^{10,13}$ The problem of the outside-in approach is how to choose the entry point and the access path through neuroforamens while removing the herniated discs with minimal bone destruction. Foraminoplasty could help access the herniated disc but may increase lumbar instability and recurrence rate attributable to damage of articular surface in facet joint. ${ }^{14}$

In this study, we proposed a far-lateral-outside-in technique for PETD to get the entry point without complex planning and facet violation. In addition, we conducted a retrospective study that compares the outcome of $\mathrm{MD}$ and PETD for the treatment of single-level symptomatic LDH.

\section{Materials and Methods}

\section{Patient Population and Demography}

From September 2017 to September 2019, all patients with symptomatic LDH who underwent either MD or PETD in the Spine Department of our institution were identified for this retrospective study. All operations were performed by three experienced spine surgeons in our department. One was responsible for microdiscectomy and the other two did PETD in this study. The inclusion criteria included patients with single level LDH between L2 and S1, clinical sciatica for at least 6 weeks, and failed attempts with non-surgical treatments. The exclusion criteria included cauda equina syndrome, progressive neurologic deficits, history of lumbar spinal surgery, modic changes in endplates, disc degeneration more than grade III by Pfirmann grading system, ${ }^{15}$ and less than 24 months of follow-up. The type of surgery used was based on the personal selection of the patient, but not on the surgeon's preference.
The patients' characteristics, age, sex, BMI, type of disc herniation, primary symptoms, and lesion level were recorded and compared. The Institutional Review Board of the Chang Gung Medical Foundation approved this study and waived the requirement for written informed consent due to the retrospective nature of the study. All procedures performed in this study complied with the ethical standards of the national research committee. The data of patients were anonymized and maintained with confidentiality.

\section{Surgical Technique}

The Far-Lateral-Outside-in Technique of PETD

Preoperative MRI was used to assess the severity, morphology, and herniation type of the lesion level (Figure 1A). PETD patients were in a prone position with local anesthetic or intravenous general anesthesia. We performed all PETD surgeries with the MaxMore spine PTED endoscopic system and the far-lateral-outside-in technique. The entry point of spinal needle was the intersection of two lines obtained from some landmarks of the patients under the assistance of fluoroscopy. One was the line of interspinous ligament drawn under the lateral view of lumbar spine ( $\mathrm{S}$ line, Figure 1B) and the other line was the inclination line (I line) based on the morphology of the herniated disc under the anterior-posterior view (Figure 1C). The spinal needle was inserted from the intersection of S line and I line on the skin along I line with a vertical angle about 10-20 degrees (Figure 1C) to the superior articular process (SAP) of the lesion level under fluoroscopic guidance. Then, a K-wire was inserted through the spinal needle and the access path through the muscle was made by dilators. The so-called Tomshidi needle was inserted through the canal and positioned with its tip on the part of SAP near the foramen (Figure 1D). After removal of Tomshidi needle, the neuroforamen was gradually expanded with rounded-blunt-end bone drills guided along the K-wire on SAP (foraminotomy, Figure 1E). After the neuroforamen was widened to $8 \mathrm{~mm}$, the endoscope could be inserted through the working sleeve, with assistance of light hammer blows if necessary. Most importantly, the working sleeve should not be located in the intervertebral disc space but behind the intervertebral disc in the epidural space under lateral view of fluoroscopy (Figure 1F). This is the key point of the far-lateral-outsidein approach. Otherwise, there is little chance of removing the sequestered parts of the intervertebral disc in the spinal canal or visualizing the nerve root. The endoscopic image can be transmitted to an external monitor by a video camera. 

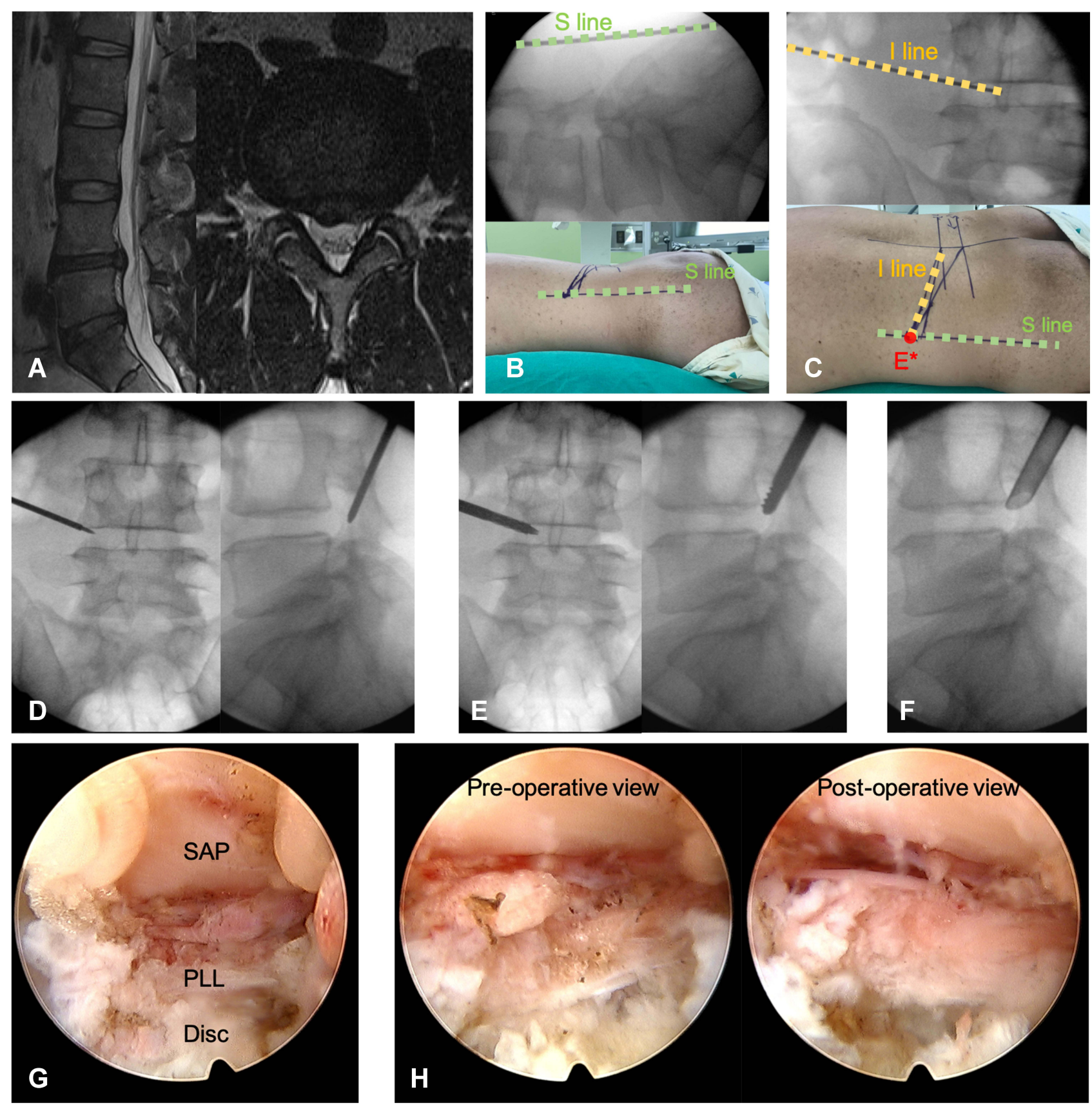

Figure I This figure was from a 38-year-old male who suffered from back pain with left leg radiation for 2 months. (A) The MRI shows an extruded disc herniation at L4-5 level with left L5 root compression. Through the assistance of fluoroscopy, the entry point $E^{*}$ for PETD surgery could be obtained from intersection of projected landmarks of (B) the interspinous ligament (S line) in lateral view and (C) the inclination line (I line) based on the morphology of the herniated disc in the anterior-posterior view. (D) The Tomshidi needle was inserted and positioned with its tip on the part of SAP near the foramen. (E) The neuroforamen was gradually expanded with rounded-blunt-end bone drills guided along the K-wire on SAP. (F) After the neuroforamen was widened to $8 \mathrm{~mm}$ (foraminotomy), the endoscope could be inserted through the working sleeve, reaching the epidural space right behind the intervertebral disc space, confirmed by lateral view of fluoroscopy. (G) After removal of blood clots and debris in endoscopic view, three layered structures were firstly recognized: SAP with flavum ligament (upper), epidural space (middle), and PLL with the herniated disc (floor). (H) Compared to preoperative endoscopic view, much looser epidural space with freely swingable nerve root under pulsation of irrigation could be visualized after the herniated disc was removed completely.

Abbreviations: S line, interspinous ligament line; I line, inclination line; E*, entry point; PETD, percutaneous endoscopic transforaminal discectomy; SAP, superior articular process; PLL, posterior longitudinal ligament.

The following layered structures should firstly be recognized (Figure 1G): SAP with flavum ligament (upper), epidural space (middle), and posterior longitudinal ligament
(PLL) with the herniated disc (lower). PLL is the natural barrier for the removal of the herniated disc, and there is less chance to injure the nerve root provided that the entire 
procedure is performed beneath it. After the sequestered, extruded or protruded disc has been completely removed, the epidural space would be loose and the nerve root should be freely visible in the endoscopic image (Figure $1 \mathrm{H}$ ).

\section{Microdiscectomy}

The patients undergoing microdiscectomy were placed in a prone position under general anesthesia. Surgical level was identified using portable (C-arm) radiography prior to the procedure. Skin, soft tissue, and paraspinal muscles were retracted by tubular expandable retractors after midline incision from posterior approach. Epidural space was exposed once hemi-laminotomy and flavum ligament resection were fulfilled on the lesion side. Herniated disc would then be removed with caution while neurologic structures are well-protected. The entire procedure was carried out using a surgical microscope with variable magnification and focalization.

\section{Surgical and Clinical Outcome}

The clinical functioning of the patients was evaluated by visual analog scale (VAS) and Oswestry Disability Index (ODI) to assess back pain, leg pain, and functional outcome. The VAS assessment was performed at pre-surgery, postoperative day 1, 3 months and 6 months. The ODI assessments were done at pre-surgery and 6-months after surgery. Blood loss, surgical time, and days of hospital stay were recorded. Regarding the outcomes, incidence of intraoperative dural tear, wound condition, recurrent leg pain, recurrent herniation, and revision surgery of the two groups were compared.

\section{Statistical Analysis}

Statistical calculations were performed using SPSS 24.0 to analyze the parameters of the patients. All quantitative variables were presented in mean standard \pm deviation and qualitative variables were shown in terms of ratios and numbers. Continuous variables were assessed by pooled Student's $t$-test. The categorical variables were performed by Fisher's exact test and Mann-Whitney test. A $\mathrm{p}$ value $<0.05$ was considered to be of statistical significance.

\section{Results}

From September 2017 to September 2019, 73 and 104 patients had PETD and MD respectively. Among those 104 MD patients, we excluded 3 patients for loss of follow up, 2 for previous lumbar surgery, 6 for discectomy more than 2 levels, 4 for being performed by other surgeons and 3 for cauda equina syndrome. In 73 PETD patients, three without complete follow-up and one with cauda equina syndrome were excluded. Therefore, 69 PETD and $86 \mathrm{MD}$ patients were enrolled in this retrospective study.

The demographic results of the 2 groups were compared. There were 40 males/29 females in PETD group and 51 males/35 females in MD group. The average age (years) was $51.25 \pm 17.26$ in PETD group and 47.26 \pm 16.49 in MD group. For body weight $(\mathrm{kg})$ and BMI $\left(\mathrm{kg} / \mathrm{m}^{2}\right)$, PETD patients were similar to MD patients. No significant difference between 2 groups in primary symptoms, the morphology of disc herniation and the lesion level was shown (Table 1).

The average surgery time (minutes) was $59.41 \pm 22.19$ in PETD group, and 69.62 \pm 18.96 in MD group. For blood loss and days of hospital stay, both of them revealed significantly better results in PETD group $(\mathrm{p}<0.001$, $\mathrm{p}=0.003$ ) (Table 2).

\section{Functional Outcomes}

There was no significant difference in preoperative ODI, VAS back pain and leg pain between the 2 groups. After surgical treatment, leg pain was much relieved in both groups at postoperative day 1, 3 months and 6 months. However, PETD group showed better improvement in VAS back pain at postoperative day 1, 3 months, 6 months and ODI at postoperative 6 months $(\mathrm{p}<0.001, \mathrm{p}<0.001$, $\mathrm{p}=0.001)$ (Table 3).

\section{Complications}

Only one patient in MD group had intraoperative dural tear. For wound condition classification, we defined deep wound infection as those who required surgical debridement for definite treatment; wound edge necrosis, discharge, skin maceration, poor closure or superficial wound infection that only required wound dressing to heal were classified as wound maceration and poor healing. There was no deep wound infection, wound maceration, or nerve root injury in PETD group; MD group had 3 patients with deep wound infection, 14 patients with wound maceration and poor healing, and none with intraoperative nerve injury $(p=0.058, p<0.001, p=1.000)$. Six PETD patients had recurrent leg pain within postoperative month 1 while there were four in MD group, mostly relieved with conservative treatment. Within first postoperative month, one PETD patient needed revision surgery due to residual herniated disc, yet three patients from 
Table I Demographic Parameters

\begin{tabular}{|c|c|c|c|}
\hline & PETD & MD & p-value \\
\hline Number of patients & 69 & 86 & \\
\hline Age & $51.25 \pm 17.26$ & $47.26 \pm 16.49$ & 0.445 \\
\hline Sex (Male: Female) & $40: 29$ & $51: 35$ & 0.871 \\
\hline Heavy worker & 13 & 16 & 0.970 \\
\hline Height $(\mathrm{cm})$ & $164.96 \pm 7.96$ & $165.39 \pm 8.97$ & 0.757 \\
\hline Body weight (kg) & $71.59 \pm 12.76$ & $72.09 \pm 16.30$ & 0.834 \\
\hline BMI $\left(\mathrm{kg} / \mathrm{m}^{2}\right)$ & $26.27 \pm 3.87$ & $26.19 \pm 4.99$ & 0.915 \\
\hline Type of disc herniation & & & 0.237 \\
\hline Protruding & 18 & 13 & \\
\hline Extruding & 14 & 20 & \\
\hline Sequestered & 37 & 53 & \\
\hline Level of disc herniation & & & 0.622 \\
\hline L2-3 & 2 & I & \\
\hline L3-4 & 7 & 5 & \\
\hline L4-5 & 36 & 50 & \\
\hline L5-SI & 24 & 30 & \\
\hline Primary symptom (leg pain) & 68 & 84 & 0.912 \\
\hline Primary symptom (back pain) & 64 & 82 & 0.756 \\
\hline Lower limb numbness & 49 & 67 & 0.356 \\
\hline Lower limb weakness & 21 & 36 & 0.180 \\
\hline Lower limb tingling sensation & 24 & 34 & 0.564 \\
\hline
\end{tabular}

Note: Values are shown in form of mean \pm standard deviation.

Abbreviations: PETLD, percutaneous endoscopic transforaminal lumbar discectomy; MD, microdiscectomy; BMI, body mass index.

Table 2 Perioperative Outcomes

\begin{tabular}{|l|c|c|c|}
\hline & PETD & MD & p-value \\
\hline Blood loss & $7.54 \pm 2.15$ & $30.47 \pm 7.01$ & $<0.001$ \\
Surgery time & $59.41 \pm 22.19$ & $69.62 \pm 18.96$ & 0.120 \\
Days of hospital stay & $1.46 \pm 0.79$ & $2.41 \pm 1.31$ & 0.003 \\
\hline
\end{tabular}

Note: Values are shown in form of mean \pm standard deviation.

Abbreviations: PETLD, percutaneous endoscopic transforaminal lumbar discectomy; MD, microdiscectomy.

MD group required debridement due to deep wound infection. Neither of the groups had surgical revision between postoperative month 1 and three months. Four PETD patients underwent revision surgeries for recurrent disc herniation between three months and one year postoperatively (Table 4). No patient in PETD group experienced devastating complications. However, one of the three patients with deep wound infection in MD group developed septic shock even after several debridements, leading to multiple organ dysfunction syndrome and expiration in two months.

\section{Discussion}

PETD has improved the surgical indications as an outside-in technique through foraminoplasty with assistance of trephines. ${ }^{16,17}$ However, it could increase the risk of intraoperative dural tear, bone surface bleeding, postoperative hematoma and damage of facet joints, leading to instability of facet joints and facet arthrosis. ${ }^{18}$ Compared with foraminoplasty, our technique provides a less invasive way to access the intra-canal space with rounded-blunt-end bone drills. We have decreased the risk of intraoperative dural tear and violation of facet joints with the use of roundedblunt-end bone drills instead of sharp trephines. Through the intersection of S line (the line of interspinous ligament) and I line (the inclination line), each spine surgeon could easily find the entry point and insert the spinal needle along I line with a vertical angle about 10-20 degrees to reach SAP. No dural tear was found in PETD patients and almost all endoscopic images were shown clearly without oozing from bone cutting surface (Figure 1G). In our opinion, the minimally invasive foraminotomy based on this far-lateral-outside-in technique accompanied with the use of rounded-blunt-end bone drills is the main reason.

Smaller wound incision, less blood loss and shorter operation time usually imply less soft tissue injury and muscle damage. Meanwhile, better recovery means early discharge and early return to work. In our study, PETD patients had better performance in VAS back pain 
Table 3 Functional Outcomes

\begin{tabular}{|l|l|c|c|c|}
\hline & & PETD & MD & P-value \\
\hline VAS back pain & Preoperative & $4.5 I \pm 1.31$ & $4.65 \pm 1.86$ & 0.475 \\
& Postoperative D I & $1.54 \pm 0.70$ & $3.63 \pm 1.02$ & $<0.00$ I \\
& Postoperative 3 M & $0.64 \pm 0.37$ & $2.16 \pm 1.92$ & $<0.00 I$ \\
VAS leg pain & Postoperative 6 M & $0.41 \pm 0.50$ & $0.74 \pm 1.62$ & 0.001 \\
& Preoperative & $7.68 \pm 0.95$ & $7.50 \pm 1.17$ & 0.298 \\
& Postoperative D I & $1.23 \pm 0.84$ & $1.27 \pm 0.64$ & 0.766 \\
ODI & Postoperative 3 M & $0.73 \pm 0.52$ & $0.92 \pm 0.78$ & 0.164 \\
& Postoperative 6 M & $0.56 \pm 0.50$ & $0.68 \pm 0.62$ & 0.196 \\
& Preoperative & $76.38 \pm 8.58$ & $75.64 \pm 8.08$ & 0.584 \\
& Postoperative 6 M & $31.23 \pm 6.59$ & $39.85 \pm 7.81$ & 0.001 \\
\hline
\end{tabular}

Note: Values are shown in form of mean \pm standard deviation.

Abbreviations: PETLD, percutaneous endoscopic transforaminal lumbar discectomy; MD, microdiscectomy; ODI, Oswestry Disability Index; VAS, visual analog scale.

Table 4 Complications

\begin{tabular}{|l|l|c|l|}
\hline & PETD & MD & P-value \\
\hline Intraoperative dural tear & 0 & 1 & 0.876 \\
Deep wound infection & 0 & 3 & 0.058 \\
Wound maceration and poor healing & 0 & $<0.00$ I \\
Nerve root injury & 0 & 0 & 1.000 \\
Recurrent leg pain within I M & 6 & 4 & 0.342 \\
Revision within I M & 1 & 3 & 0.629 \\
Revision between I -3 M & 0 & 0 & 1.000 \\
Revision between 3 M - I Y & 4 & 0 & 0.02 I \\
Recurrent herniation at final F/U & 4 & I & 0.049 \\
\hline
\end{tabular}

Notes: Values are shown in form of mean \pm standard deviation. Deep wound infection: surgical debridement. Wound maceration and poor healing: wound edge necrosis, discharge, skin maceration, poor closure and superficial wound infection.

Abbreviations: PETLD, percutaneous endoscopic transforaminal lumbar discectomy; MD, microdiscectomy; F/U, follow-up.

postoperatively, and better ODI than MD patients at 6 months postoperatively. Less postoperative back pain and soft tissue scarring are major causes for better functional outcome. $^{19,20}$ Unlike open microdiscectomy, PETD is a minimally invasive treatment with no paraspinal muscle detached, no bony destruction and less epidural scarring. This could make a difference in effectiveness, leading to faster rehabilitation and integration, and thus better functional prognosis with lower costs to society. ${ }^{21}$ As implied in the comparative cohort study with a 5-year follow-up, Ahn et al found PETD had shorter operation time, hospital stay, recovery time, and time of returning to work. ${ }^{20}$ This was most likely because MD caused more iatrogenic damage to the surrounding tissue compared to PETD. With the advanced endoscopic tools and a skillful surgeon, PETD can achieve a better short-term outcome in the alleviation of postoperative discomfort.

Microdiscectomy is still one kind of open surgery, and wound problem is one of the main complications. ${ }^{22}$
According to a systematic review, microdiscectomy had an overall wound complication rate between $1.2-2.1 \%$, which was higher than percutaneous discectomy. ${ }^{23} \mathrm{Li}$ et al drew the conclusion with the same tendency in their comparative cohort study. ${ }^{24}$ Our study showed that MD had a higher overall wound complication rate: $19.8 \%$ with $3.5 \%$ deep wound infection and $16.3 \%$ delayed wound healing. Although the deep wound infection rate of $3.5 \%$ is slightly above average, our major concern is that 14 out of $86 \mathrm{MD}$ patients had delayed wound healing with maceration for 1-2 weeks. It would affect the effectiveness of surgical prognosis, patient's satisfaction, postoperative back pain and return to work. Wound problems usually result in significant increase in morbidity and incur a substantial cost to the health care system. ${ }^{25}$ One study proved that each episode of wound infection following a spine procedure contributed to a mean increase in the cost of care by $\$ 4067 .{ }^{26}$ Although the deep wound infection rate of $3.5 \%$ was not actually of statistical significance, those three patients in our study suffered 
repeated debridement and prolonged antibiotics treatment. One of them expired owing to devastating complications during hospitalization.

In our study, 10 patients in total had recurrent leg discomfort within postoperative month 1 (6 in PETD, $8.69 \%$ and 4 in MD, 4.65\%). Only one of the 69 PETD patients had poor response to conservative treatment and received revision surgery due to residual disc fragments. This patient was included in the early stage of our study during the learning curve of PETD surgeons. PETD is a type of minimal and limited discectomy expected to exhibit a high recurrence rate. Even with the advanced endoscopic camera and variation of methods to remove herniated disc lesions, PETD still has difficulty gaining a complete view of surgical site, especially for patients with smaller foraminal space or severe disc degeneration. However, recent studies demonstrated that the recurrence rate for PETD was comparable to that of open discectomy. ${ }^{27,28}$ Although residual disc fragments were found in $2.8-15 \%$ of patients after PETD $^{29,30}$ and the persistent compression of nerve roots is a cause of reoperation, not all patients with residual disc fragments are symptomatic. Therefore, most surgeons suggest a "watchful waiting" strategy for asymptomatic patients, and try conservative treatment first for those with minor symptoms. $^{31}$

Recurrent LDH has been defined as repeat disc herniation at the same level with MRI confirmation after an initial period of symptomatic relief. The recurrence rate after surgical treatment has been reported as 5-15\%. ${ }^{32,33}$ Symptomatic recurrent $\mathrm{LDH}$ is considered as one of the major complications of PETD, and the risk factors for early recurrence after PETD are BMI, disc degeneration scale, combined herniated nucleus pulposus, and early ambulation. ${ }^{34}$ In our study, the recurrent LDH rate of PETD patients was $5.79 \%$, higher than that of the MD patients. Because of no significant difference in BMI, age, sex, and disc morphology, early ambulation and quick functional recovery might be accountable for the higher recurrence rate in PETD group. Early discharge, immediate back pain relief and higher ODI help PETD patients to return to work and even high level of activities quickly after the surgeries.

To the best of our knowledge, our study is the first to propose the surgical technique of PETD, making the complicated simple, transforming 3-dimensional endoscopic trajectory and entry point into a brief 2-dimensional method via the intersection of just two lines. Surgeons could improve their learning curve and decrease intraoperative complications by referring to our far-lateraloutside-in technique. We also detailed postoperative wound problems of microdiscectomy and short-term higher recurrent rate of PETD to explore features of those two surgical operations. Under adequate surgical indications and techniques, PETD could result in higher functional outcome with less wound problems.

There are some limitations to this study. To begin with, this was a retrospective study with non-randomized patient samples, which is prone to selection bias. Then, performance bias may exist due to different surgery types performed by 3 surgeons. To decrease the influence of performance bias, we allocated surgery types by surgeon specialty and set up the same inclusion and exclusion criteria, data collection methods, outcome determination, and follow-up time. Furthermore, the choice of surgical method was determined purely by the patients' preference. As a result, more future studies will be necessary to fully assess the clinical outcome.

\section{Conclusion}

$\mathrm{MD}$ has been the gold standard procedure for LDH. However, with the advanced endoscopic technology and surgical techniques, PETD has emerged as an alternative method due to higher functional outcome and less wound problems. While both methods demonstrated overall promising sciatica relief, further studies are needed to compare the clinical efficacy and prognosis more thoroughly.

\section{Disclosure}

The authors report no conflicts of interest in this work.

\section{References}

1. Yang H, Liu H, Li Z, et al. Low back pain associated with lumbar disc herniation: role of moderately degenerative disc and annulus fibrous tears. Int J Clin Exp Med. 2015;8(2):1634-1644.

2. Konstantinou K, Dunn KM. Sciatica: review of epidemiological studies and prevalence estimates. Spine. 2008;33(22):2464-2472. doi:10.1097/BRS.0b013e318183a4a2

3. Mikkonen P, Heikkala E, Paananen M, et al. Accumulation of psychosocial and lifestyle factors and risk of low back pain in adolescence: a cohort study. Eur Spine J. 2016;25(2):635-642. doi:10.1007/s00586015-4065-0

4. Gautschi OP, Hildebrandt G, Cadosch D. [Acute low back painassessment and management]. Praxis. 2008;97(2):58-68. German. doi:10.1024/1661-8157.97.2.58

5. Gibson JN, Waddell G. Surgical interventions for lumbar disc prolapse: updated Cochrane Review. Spine. 2007;32(16):1735-1747. doi:10.1097/BRS.0b013e3180bc2431 
6. Liu T, Zhou Y, Wang J, et al. Clinical efficacy of three different minimally invasive procedures for far lateral lumbar disc herniation. Chin Med J. 2012;125(6):1082-1088.

7. Lau D, Han SJ, Lee JG, Lu DC, Chou D. Minimally invasive compared to open microdiscectomy for lumbar disc herniation. J Clin Neurosci. 2011;18(1):81-84. doi:10.1016/j.jocn.2010.04.040

8. Kambin P, Sampson S. Posterolateral percutaneous suction-excision of herniated lumbar intervertebral discs. Report of interim results. Clin Orthop Relat Res. 1986;1(207):37-43.

9. Ahn Y, Lee SH. Outcome predictors of percutaneous endoscopic lumbar discectomy and thermal annuloplasty for discogenic low back pain. Acta Neurochir. 2010;152(10):1695-1702. doi:10.1007/ s00701-010-0726-2

10. Kambin P, Gellman H. Percutaneous lateral discectomy of the lumbar spine a preliminary report. Clin Orthop Relat Res. 1983;174:127-132.

11. Yeung AT. Minimally invasive disc surgery with the Yeung Endoscopic Spine System (YESS). Surg Technol Int. 1999;8:267-277.

12. Ruetten S, Komp M, Godolias G. An extreme lateral access for the surgery of lumbar disc herniations inside the spinal canal using the full-endoscopic uniportal transforaminal approach-technique and prospective results of 463 patients. Spine. 2005;30(22):2570-2578. doi:10.1097/01.brs.0000186327.21435.cc

13. Yeung AT, Tsou PM. Posterolateral endoscopic excision for lumbar disc herniation: surgical technique, outcome, and complications in 307 consecutive cases. Spine. 2002;27(7):722-731. doi:10.1097/ 00007632-200204010-00009

14. Qiao P, Xu T, Zhang W, Fang Z, Ding W, Tian R. Foraminoplasty affects the clinical outcomes of discectomy during percutaneous transforaminal endoscopy: a two-year follow-up retrospective study on 64 patients. Int J Neurosci. 2020;131:1-6.

15. Griffith JF, Wang YX, Antonio GE, et al. Modified Pfirrmann grading system for lumbar intervertebral disc degeneration. Spine. 2007;32 (24):E708-712. doi:10.1097/BRS.0b013e31815a59a0

16. Tsou PM, Yeung AT. Transforaminal endoscopic decompression for radiculopathy secondary to intracanal noncontained lumbar disc herniations: outcome and technique. Spine J. 2002;2(1):41-48. doi:10.1016/S1529-9430(01)00153-X

17. Yue JJ, Long W. Full endoscopic spinal surgery techniques: advancements, indications, and outcomes. Int J Spine Surg. 2015;9:17. doi:10.14444/2017

18. Sairyo K, Sakai T, Higashino K, Inoue M, Yasui N, Dezawa A. Complications of endoscopic lumbar decompression surgery. Minim Invasive Neurosurg. 2010;53(4):175-178. doi:10.1055/s-00301262814

19. Singh R, Zeng Xin G, Hirachan MP, Yu Cheng L. Outcome of percutaneous transforaminal endoscopic lumbar surgery in $>60$-yearold patients with low back pain. Asian Spine J. 2018;12(3):511-517. doi:10.4184/asj.2018.12.3.511

20. Ahn Y, Lee SG, Son S, Keum HJ. Transforaminal endoscopic lumbar discectomy versus open lumbar microdiscectomy: a comparative cohort study with a 5-year follow-up. Pain Physician. 2019;22 (3):295-304. doi:10.36076/ppj/2019.22.295

Journal of Pain Research

\section{Publish your work in this journal}

The Journal of Pain Research is an international, peer reviewed, open access, online journal that welcomes laboratory and clinical findings in the fields of pain research and the prevention and management of pain. Original research, reviews, symposium reports, hypothesis formation and commentaries are all considered for publication. The manuscript

Submit your manuscript here: https://www.dovepress.com/journal-of-pain-research-journal
21. Gadjradj PS, Tulder M, Dirven CMF, Peul WC, Harhangi BS. Clinical outcomes after percutaneous transforaminal endoscopic discectomy for lumbar disc herniation: a prospective case series. Neurosurg Focus. 2016;40(2):E3. doi:10.3171/2015.10. FOCUS15484

22. Shriver MF, Xie JJ, Tye EY, et al. Lumbar microdiscectomy complication rates: a systematic review and meta-analysis. Neurosurg Focus. 2015;39(4):E6. doi:10.3171/2015.7.FOCUS15281

23. Michael FS, Jack JX, Erik YT, et al. Lumbar microdiscectomy complication rates: a systematic review and meta-analysis. Neurosurg Focus. 2015;39(4):E6.

24. Li Z, Zhang C, Chen W, et al. Percutaneous endoscopic transforaminal discectomy versus conventional open lumbar discectomy for upper lumbar disc herniation: a comparative cohort study. Biomed Res Int. 2020;2020:1852070. doi:10.1155/2020/1852070

25. Calderone RR, Garland DE, Capen DA, Oster H. Cost of medical care for postoperative spinal infections. Orthop Clin North Am. 1996;27(1):171-182. doi:10.1016/S0030-5898(20)32060-5

26. Whitmore RG, Stephen J, Stein SC, et al. Patient comorbidities and complications after spinal surgery: a societal-based cost analysis. Spine. 2012;37(12):1065-1071. doi:10.1097/ BRS.0b013e31823da22d

27. Kim CH, Chung CK, Park CS, Choi B, Kim MJ, Park BJ. Reoperation rate after surgery for lumbar herniated intervertebral disc disease: nationwide cohort study. Spine. 2013;38(7):581-590. doi:10.1097/BRS.0b013e318274f9a7

28. Kim CH, Chung CK, Choi Y, et al. The selection of open or percutaneous endoscopic lumbar discectomy according to an age cut-off point: nationwide cohort study. Spine. 2015;40(19):E1063-1070. doi:10.1097/BRS.0000000000001053

29. Choi KC, Lee JH, Kim JS, et al. Unsuccessful percutaneous endoscopic lumbar discectomy: a single-center experience of 10,228 cases. Neurosurgery. 2015;76(4):372-380; discussion 380-371; quiz 381. doi: $10.1227 / \mathrm{NEU} .0000000000000628$

30. Heo JH, Kim CH, Chung CK, et al. Quantity of disc removal and radiological outcomes of percutaneous endoscopic lumbar discectomy. Pain Physician. 2017;20(5):E737-e746.

31. Baek J, Yang SH, Kim CH, et al. Postoperative longitudinal outcomes in patients with residual disc fragments after percutaneous endoscopic lumbar discectomy. Pain Physician. 2018;21(4):E457E466.

32. Kim JM, Lee SH, Ahn Y, Yoon DH, Lee CD, Lim ST. Recurrence after successful percutaneous endoscopic lumbar discectomy. Minim Invasive Neurosurg. 2007;50(2):82-85. doi:10.1055/s-2007-982504

33. El Shazly AA, El Wardany MA, Morsi AM. Recurrent lumbar disc herniation: a prospective comparative study of three surgical management procedures. Asian $J$ Neurosurg. 2013;8(3):139-146. doi:10.4103/1793-5482.121685

34. Kim HS, You JD, Ju CI. Predictive scoring and risk factors of early recurrence after percutaneous endoscopic lumbar discectomy. Biomed Res Int. 2019;2019:6492675. doi:10.1155/2019/6492675

management system is completely online and includes a very quick and fair peer-review system, which is all easy to use. Visit http:// www.dovepress.com/testimonials.php to read real quotes from published authors. 